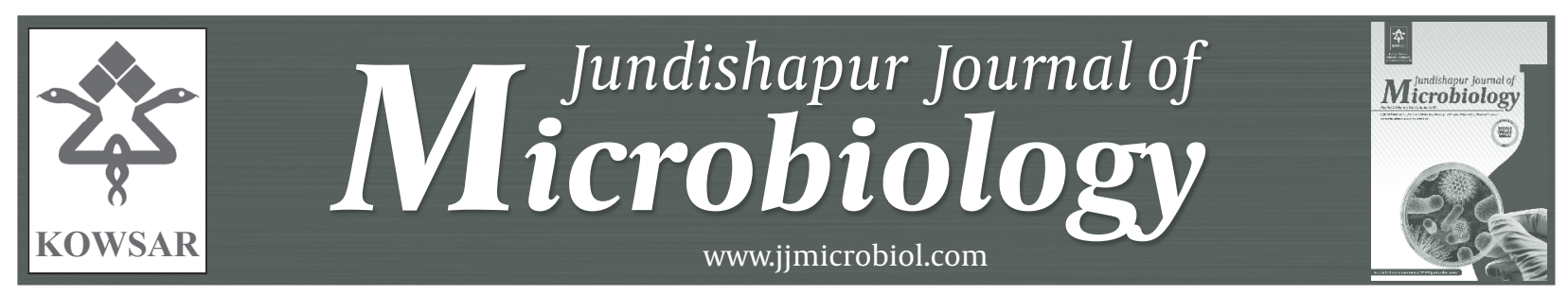

\title{
Multi-Drug Resistant Acinetobacter-Derived Cephalosporinase and OXAs- etC Genes in Clinical Specimens of Acinetobacter spp. Isolated From Teach- ing Hospital
}

\author{
Reza Khaltabadi Farahani ${ }^{1}$, Rezvan Moniri ${ }^{1,2^{*}}$, Kamran Dastehgoli $^{2}$ \\ ${ }^{1}$ Department of Microbiology and Immunology, Faculty of Medicine, Kashan University of Medical Sciences, Kashan, IR Iran \\ ${ }^{2}$ Anatomical Sciences Research Center, Kashan University of Medical Sciences, Kashan, IR Iran \\ * Corresponding author: Rezvan Moniri, Department of Microbiology and Immunology, Faculty of Medicine, Kashan University of Medical Sciences, Kashan, \\ IR Iran.Tel.: +98-3615550021-25 Ext. 539, Fax: +98-3615551112, E-mail: moniri@kaums.ac.ir
}

\begin{abstract}
A B S T R A C T
Background: Hospital-acquired infections caused by multi-drug resistant Acinetobacter spp. are often extremely difficult to treatand this has proved to be a serious problem worldwide.

objectives: The aim of this study was to determine the incidence rates and distribution patterns of multi-drug resistant (MDR) Acinetobacter spp. strains and the occurrence of Acinetobacter-derived cephalosporinase (ADC-7) and OXA-type carbapenemases (OXAsetC genes) in clinical specimens in the Beheshti Teaching Hospital in Kashan, Iran.

Materials and Methods: This descriptive study was carried out on sixty isolates of Acinetobacter spp. and clinical samples collected from patients. The level of antibiotic resistance was determined by the disc diffusion method and the results were interpreted according to the Clinical and Laboratory Standards Institute (CLSI) procedure. Polymerase chain reaction (PCR) amplification of the genetic determinants of resistance was also determined.

Results: The resistance rates were; amikacin (80\%), tobramycin (68.3\%), ceftazidime (60\%), ciprofloxacin (55\%), piperacillin/tazobactam (51.7\%), doxycycline (50\%), SXT/TMP (sulfamethoxazole / trimethoprim)(48.3\%), levofloxacin (43.3\%), gentamicin (40\%), imipenem (25\%), and sulbactam/ampicillin (20\%). The frequency of MDR Acinetobacter spp. strains isolated was found to be 56.7\%. These isolates were most sensitive to imipenem followed by ampicillin/sulbactam and gentamicin. The prevalence of genes for ADC-7 and OXAsetC in the Acinetobacter spp. was; 34 (56.7\%) and 32 (53.3\%), respectively. The positive percentages of MDR isolates for ADC-7 were $82.4 \%$ and for OXAsetC they were $73.5 \%$.

Conclusions: Our phenotypic analysis demonstrated that Acinetobacter spp. isolates were resistant to most clinically significant antibiotic classes. This is the first report concerning Acinetobacter-derived cephalosporinase, blaADC, enzymes in Acinetobacter spp. isolates from Iran.
\end{abstract}

Keywords: Acinetobacter spp.; Acinetobacter-Derived Cephalosporinase; OXA-Type Carbapenemases; Antibiotic Susceptibility; Iran

Copyright ( () 2013, Kowsar Corp.; Published by Kowsar Corp.

Article type: Research Article; Received: 15 Apr 2012, Revised: 27 May 2012, Accepted: 07 Jun 2012; DOI: 10.5812/jjm.5059

-Implication for health policy/practice/research/medical education:

The emergence and rapid spread of multidrug-resistant (MDR) isolates of Acinetobacter spp. causing nosocomial infections are of great concern worldwide.

Please cite this paper as:

Khaltabadi Farahani R, Moniri R, Dastehgoli K. Multi-Drug ResistantAcinetobacter-Derived Cephalosporinase and OXAsetC Genes in Clinical Specimens of Acinetobacter spp. Isolated From Teaching Hospital.JundishapurJ Microbiol. 2013;6(2):181-185. DOI:10.5812/jjm.5059

Copyright (C) 2013 Ahvaz Jundishapur University of Medical Sciences; Published by Kowsar Corp.

This is an Open Access article distributed under the terms of the Creative Commons Attribution License (http://creativecommons.org/licenses/by/3.0), which permits unrestricted use, distribution, and reproduction in any medium, provided the original work is properly cited. 


\section{Background}

Acinetobacter spp. have emerged as important opportunistic pathogens in hospitals and these are often related to international nosocomial outbreaks (1). These infections are complicated to treat due to the occurrence of multidrug-resistant (MDR) organisms, which include resistance to; beta-lactams, aminoglycosides, fluoroquinolones and more recently, carbapenems (2). There has been increasing reports of carbapenem resistance in Acinetobacter strains worldwide (1). Increases in the prevalence of resistant strains has also been seen, which has compromised patient treatment with; aminoglycosides, penicillins, extended spectrum cephalosporins, and more recently, fluoroquinolones (1-3).

The principal mechanism of carbapenem resistance is enzymatic hydrolysis mediated by carbapenemases. In $A$ baumannii these enzymes are usually OXA-type carbapenemases, which are members of class D according to the Ambler classification system (4). The OXA-type carbapenemases are divided into five sub-groups, four of these are acquired carbapenemases and their nomination is according to the circulation of genes blaOXA in different geographic areas. Five main phylogenetic subgroups of OXA-type carbapenemases have been recognized in $A$. baumannii; OXA-23-like, OXA-40-like, OXA-51-like, OXA-58like, and OXA-143-like (4).

The major subgroup is OXA-51-like, which is responsible for chromosomal encoded enzymes, thus the natural existence of OXA-type carbapenemases in A. baumannii is known worldwide (4). Here, we report an analysis of the antibiotic susceptibility profile and genetic determinants of antibiotic resistance in 60 isolates of Acinetobacter spp. obtained from patients in our teaching hospital.

\section{Objectives}

The aims of the present study were to determine the frequency of MDR and the occurrence of ADC-7 and OXAsetC genes of Acinetobacter spp. from patients hospitalized in a tertiary teaching hospital.

\section{Materials and Methods}

A total of 60 Acinetobacter spp. clinical isolates were collected during January 2010 to September 2010 from the Beheshti Hospital in Kashan, Iran. Conventional biochemical tests such as; glucose, xylose, lactose, indole, ONPG
(nitrophenyl-beta-D-galactopyranoside), fermentation of manitol and maltose, motility and production of $\mathrm{H} 2 \mathrm{~S}$, were used for the identification of isolated Acinetobacter spp. at the species level in 60 Gram negative, short rods showing both a negative reaction on oxidase testing and the lack of lactose fermentation. A. baumannii is saccharolytic, and A. lwoffii is asaccharolytic. The strains were isolated from; blood (58.4\%), sputum (16.7\%), urine (13.3\%), cerebrospinal fluids (8.3\%), and pleural fluid (3.3\%) samples.

Antimicrobial susceptibility testing was performed on all 60 isolates according to the standard method established

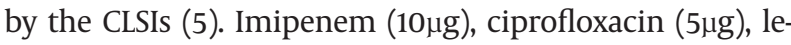

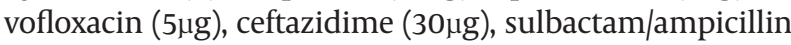

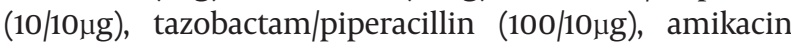

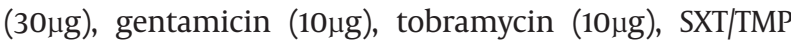
$(1.25 / 23.75 \mu \mathrm{g})$, doxycycline $(30 \mu \mathrm{g})$, and minocycline $(30 \mu \mathrm{g})$ disks (Becton Cockeysville, USA, Microbiology Systems ) were used. Escherichia coli ATCC 25922 and Pseudomonas aeruginosa ATCC 27853 were used as quality controls in each susceptibility test. Multidrug resistance was defined in this analysis as resistance to three or more representatives of the following classes of antibiotics; quinolones (ciprofloxacin, levofloxacin), broad-spectrum cephalosporins (ceftazidime), beta-lactamase inhibitor/beta-lactams (sulbactam/ ampicillin, tazobactam/piperacillin), aminoglycosides (amikacin, gentamicin and tobramycin), tetracyclines (doxycycline, minocycline), trimethoprim-sulfamethoxazole and carbapenems (imipenem).

All objective genes and related primers used for polymerase chain reaction (PCR) amplification are listed in Table 1 . The primers used for these tests were purchased from the Cinagene Company, Iran. For PCR, a 1:10 dilution of 24 hours culture was boiled for 10 min., then amplification was performed with 1:10 of this dilution as the DNA template. PCR conditions included 30 cycles of amplification under the following conditions: Denaturation at $95^{\circ} \mathrm{C}$ for $30 \mathrm{sec}$, annealing at $50^{\circ} \mathrm{C}$ for $30 \mathrm{sec}$., and then at $40^{\circ} \mathrm{C}$ for 40 sec., cycling was followed by a final extension at $72^{\circ} \mathrm{C}$ for $30 \mathrm{~min}$. PCR products were set on $2.0 \%$ agarose gels, stained with ethidium bromide, and photo-graphed by UV illumination (Ingenius, Syngene). The 100-bp DNA ladder (Bioneer, Korea) was used to assess the PCR product size. The antimicrobial resistance rates of the isolates were compared using a chi-square test or Fisher's exact test with SPSS version 16 for Windows (SPSS, Chicago, IL).

Table 1. Primers for Amplification of Genes From Acinetobacter spp. Isolates.

\begin{tabular}{|c|c|c|c|c|}
\hline Primer Name & Primer Sequence ( 5 ' to 3 ') & $\begin{array}{l}\text { Annealing Tem- } \\
\text { perature }\left({ }^{\circ} \mathrm{C}\right)\end{array}$ & Target Gene(s) & Reference \\
\hline ADC-7 FOR & ATGCGATTTAAAAAAATTTCTTGT & \multirow[t]{2}{*}{50} & \multirow{2}{*}{$\begin{array}{l}\text { blaADC-1,blaADC-2,blaADC-3,blaADC- } \\
\text { 4,blaADC-5,blaADC-6,blaADC-7 }\end{array}$} & \multirow[t]{2}{*}{ (6) } \\
\hline ADC-7 REV & TTATTTCTTTATTGCATTCAG & & & \\
\hline OXA SET C FOR & ACAGAARTATTTAAGTGGG & \multirow[t]{2}{*}{47} & \multirow{2}{*}{$\begin{array}{l}\text { blaOXА-51,blaOXА-58,blaOXА-64,blaOXА- } \\
\text { 69,blaOXA-70,blaOXA-71,blaOXA-75,blaOXA-78 }\end{array}$} & \multirow[t]{2}{*}{ (7) } \\
\hline OXA SET C REV & GGTCTACAКССMWTCCССА & & & \\
\hline
\end{tabular}




\section{Results}

Acinetobacter spp. isolates were recovered from 60 patients including, 35 men (58.3\%) and 25 women (41.7\%). From 60 Acinetobacter spp. isolates in the present study, 48 (80\%) were recognized as A. baumannii, $10 \%$ as A. lwoffii and $10 \%$ as other genomic species of Acinetobacter. The characteristics of the patients are summarized in Table 2 .

Table 2. Demographic Characteristics of the Study Population.

\begin{tabular}{ll}
\hline Parameters & Patients, No. (\%) a,b \\
\hline Age, $\mathbf{y}<\mathbf{4 0}$ & $29(48.3)$ \\
\hline Age, $\mathbf{y}>\mathbf{4 0}$ & $31(51.7)$ \\
Sex (male/female) & $35 / 25(58.3 / 41.7)$ \\
\hline Hospital Sampling Ward & \\
Emergency room & $24(40)$ \\
Internal medicine & $15(25)$ \\
ICU & $15(25)$ \\
Pediatrics & $6(10)$ \\
\hline $\begin{array}{l}\text { a range[minimum, maximum]: } 81[4,85] \\
\text { b Mean } \pm \text { SD: } 39.27 \pm 19.20\end{array}$
\end{tabular}

The mean age for the studied population was 39.3 years ( \pm 19.2$)$, with a range of 4 to 85 years. Table 3 summarizes the isolation sites and antibiotic resistance patterns in this study. The resistance pattern of Acinetobacter spp. isolates in our hospital showed a very high resistance rate to amikacin (80\%) and tobramycin (68.3\%). Isolated Acinetobacter spp. were more sensitive to ampicillin/sulbactam than to the other antibiotics tested. From the 60 isolates, 34 (56.7\%) were resistant to at least three classes of antibiotics and classified as multi-drug resistant (MDR). Surprisingly, among the MDR isolates $97.1 \%$ were resistant to amikacin, $91.2 \%$ to ceftazidime, $85.3 \%$ to piperacillin/tazobactam, $82.4 \%$ to ciprofloxacin, $73.5 \%$ to levofloxacin and trimethoprim/ sulfamethoxazole, $70.6 \%$ to doxycycline and gentamicin, $67.6 \%$ to minocycline, $58.8 \%$ to tobramycin, $44.1 \%$ to imipenem, and 35.3\% to ampicillin/sulbactam.

From the hospital departments and type of specimens the following levels of MDR were found; $83.3 \%$ (5/6) of the isolates were from pediatrics, and $80 \%$ (12/15) were from ICU, 90\% (9/10) from sputum samples and $40 \%$ (14/35) of the blood isolates were MDR. The percentage of antibiotic resistance genes detected in Acinetobacter spp. strains according to the hospital locations are shown in Table 4. The frequency rates for ADC-7 and OXAsetC in ICU isolates were higher than that found in the other wards. The percentage rates of antibiotic sensitivity and resistance patterns of Acinetobacter spp. isolates in relation to the genes encoding beta-lactamases detected, are shown in $\mathrm{Ta}$ - ble 5 . More than $80 \%$ of the OXA-type carbapenemases positive isolates were resistant to amikacin. The highest resistance rate of ampC Acinetobacter-derived cephalosporinase positive isolates were observed in ceftazidime and amikacin, respectively. The ampC Acinetobacter-derived cephalosporinase, blaADC, was detected in $57.6 \%$ of the stains by PCR amplification. Thirty strains from a total of $34(88.2 \%)$ blaADC positive strains were resistant to ceftazidime and 28 out of $34(82.4 \%)$ were MDR. By using PCR, a blaOXA69-like gene was found in $53.3 \%$ of the isolates, and 25 out of 32 strains (78.1\%) were resistant to ceftazidime and MDR, simultaneously.

\section{Discussion}

Acinetobacter spp. has emerged as a major pathogen of nosocomial infections, while the management of this pathogen, due to the increased emergence of carbapenem-resistant strains, has become a significant challenge (3). Its low level susceptibility to ceftazidime $(40 \%)$ is very important. This may be the consequence of an enhanced production of class- $C$ chromosomal beta-lactamase $(A m p C)$, which is possibly related to the extensive use of cephalosporin antibiotics in our hospital. Overall, piperacillin/tazobactam with a $48.3 \%$ susceptibility rate is the best treatment choice. Imipenem with a $75 \%$ susceptibility rate, exhibited the best in vitro antimicrobial action compared to the other beta-lactamases. Susceptibility to imipenem varies around the world. Previous reports indicated approximately; 51\% imipenem-sensitive isolates in Iran (8), 43.4\% to 56.7\% in Turkey (9), and $10 \%$ to 55\% in Saudi Arabia (8). Imipenem-resistant strains were analyzed for their genetic determinants. Sixty percent of them were positive for blaOXA-51-like gene and $80 \%$ for ADC-7 genes.

It has been shown that the majority of the carbapenem resistance in A. baumannii is due to the production of carbapenemases, especially those belonging to the carbapenem-hydrolyzing class D beta-lactamases, which are encoded by the blaOXA-51-like, blaOXA23-like, blaOXA-24-like, and blaOXA-58-like genes (3). The blaOXA-51-like gene is native and was originally located on the chromosome of A. baumannii. It is chromosomally positioned and is extensively prevalent. Similar to other class of D enzymes, they have a better attraction for imipenem than meropenem. Their role in carbapenem resistance is related to the presence of an insertion sequence ISAba1, situated upstream, possibly providing a promoter for the hyperproduction of beta-lactamase genes $(3,10,11)$. A wide diversity of OXA genes exist among the strains of A. baumannii isolated in Iran (8). A high incidence of OXA-type carbapenemase in MDR A. baumannii has been reported in the northwest of Iran (12). 
Table 3. Frequency of Antibiotic Resistance Patterns of 60 Acinetobacter spp. Isolates in Relation to Various Specimens From Hospitalized Patients

\begin{tabular}{|c|c|c|c|c|c|c|c|c|c|c|c|c|c|}
\hline & 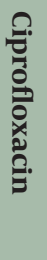 & 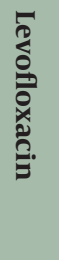 & 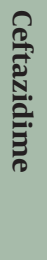 & 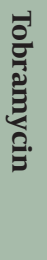 & 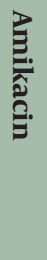 & 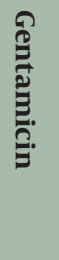 & 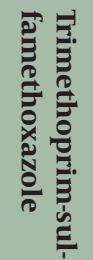 & 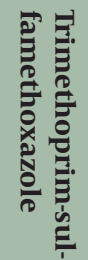 & 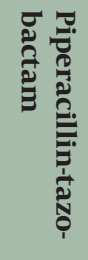 & 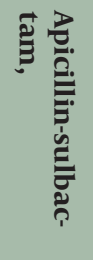 & 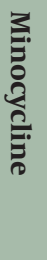 & 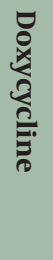 & 夛 \\
\hline Blood stream & 15 & 9 & 16 & 25 & 27 & 8 & 12 & 8 & 12 & 5 & 14 & 17 & 14 \\
\hline Cerebrospinal fluid & 3 & 4 & 3 & 4 & 5 & 3 & 4 & 1 & 3 & 3 & 3 & 3 & 3 \\
\hline Urine & 5 & 4 & 5 & 6 & 5 & 4 & 6 & 4 & 5 & 2 & 5 & 6 & 6 \\
\hline Pleural fluid & 2 & 1 & 2 & 1 & 2 & 1 & 1 & 0 & 2 & 0 & 1 & 1 & 2 \\
\hline Sputum & 8 & 8 & 10 & 5 & 9 & 8 & 6 & 2 & 9 & 2 & 6 & 3 & 9 \\
\hline Total & 33 & 26 & 36 & 41 & 48 & 24 & 29 & 15 & 31 & 12 & 29 & 30 & 34 \\
\hline
\end{tabular}

Table 4. Percentage Rate of Antibiotic Resistant Genes Detected in Sixty Isolated Acinetobacter spp. According to Hospital Location.

\begin{tabular}{llllll}
\hline $\begin{array}{l}\text { Genes Encoding } \\
\text { b-Lactamases }\end{array}$ & $\begin{array}{l}\text { Gene Detection in } \\
\text { Isolates, No. }(\%)\end{array}$ & ICU & Emergency Room & Pediatrics & Internal Medicine \\
\hline ADC-7 & $34(56.7)$ & $13(38.2)$ & $6(17.6)$ & $4(11.8)$ & $11(32.4)$ \\
\hline OXAset C & $32(53.3)$ & $12(37.5)$ & $8(25)$ & $4(12.5)$ & $8(25)$ \\
\hline
\end{tabular}

a OXAsetC: includes blaOXA-51-, blaOXA-58-, blaOXA-64-, blaOXA-69-, blaOXA-70-, blaOXA-71-, blaOXA-75- and blaOXA-78-like genes.

Table 5. Percentage Rates of Antibiotic Sensitivity and Resistance Patterns Detected in Sixty Acinetobacter spp. Isolates in Relation to Genes Encoding $\beta$-Lactamases.

\begin{tabular}{|lllllll}
\hline & \multicolumn{3}{c}{ OXA Set C Positive } & \multicolumn{3}{c}{ ADC-7 Positive } \\
\hline & $\mathrm{S}$ & $\mathrm{I}$ & $\mathrm{R}$ & $\mathrm{S}$ & $\mathrm{I}$ & $\mathrm{R}$ \\
\hline Amikacin $(\mathbf{3 0} \mu \mathbf{g})$ & 9.3 & 9.4 & 81.3 & 8.8 & 5.9 & 85.3 \\
\hline Gentamicin $(\mathbf{1 0} \mu \mathbf{g})$ & 31.2 & 0 & 68.8 & 29.4 & 0 & 70.6 \\
\hline Tobramycin $(\mathbf{1 0} \mu \mathbf{g})$ & 15.6 & 37.5 & 46.9 & 14.7 & 35.3 & 50 \\
\hline Tazobactam/ Piperacillin $(\mathbf{1 0 0} / \mathbf{1 0} \mu \mathbf{g})$ & 18.8 & 6.2 & 75 & 14.7 & 8.8 & 76.5 \\
\hline Sulbactam/Ampicillin $(\mathbf{1 0} / \mathbf{1 0} \mu \mathbf{g})$ & 46.9 & 28.1 & 25 & 28.2 & 29.4 & 32.4 \\
\hline Ceftazidime $(\mathbf{3 0} \mu \mathbf{g})$ & 12.5 & 9.4 & 78.1 & 5.9 & 5.9 & 88.2 \\
\hline Ciprofloxacin $(\mathbf{5} \mu \mathbf{g})$ & 6.2 & 21.9 & 71.9 & 11.8 & 14.7 & 73.5 \\
\hline Levofloxacin $(\mathbf{5} \mu \mathbf{g})$ & 28.1 & 0 & 71.9 & 29.4 & 0 & 70.6 \\
\hline Imipenem $(\mathbf{1 0} \mu \mathbf{g})$ & 68.8 & 3.1 & 28.1 & 61.8 & 2.9 & 35.3 \\
\hline Doxycycline $(\mathbf{3 0} \mu \mathbf{g})$ & 12.5 & 34.4 & 53.1 & 8.8 & 29.4 & 61.8 \\
\hline Minocycline $(\mathbf{3 0} \mu \mathbf{g})$ & 9.4 & 25 & 65.6 & 8.8 & 20.6 & 70.6 \\
\hline SXT/TMP $(\mathbf{1 . 2 5} / \mathbf{2 3 . 7 5} \mu \mathbf{g})$ & 12.5 & 25 & 62.5 & 12.5 & 25 & 62.5 \\
\hline
\end{tabular}

Abbreviations: S, sensitive; I, intermediate; R, resistance

Chromosomal-encoded blaOXA genes play a challenging role in the antibiotic resistance of $A$. baumannii (13). The blaOXA-51-like genes are omnipresent in A. baumannii. Insertion of ISAba1 upstream from blaOXA-51-like provides a strong promoter, resulting in enhanced gene expression and carbapenem resistance $(14,15)$. Chromo- somally encoded Acinetobacter-derived cephalosporinases (ADCs) are intrinsic in all A. baumannii strains. The presence of ISAba1 elements are highly associated with increased $A m p C$ gene expression and resistance to extended-spectrum cephalosporins (16). More than 60\% of ADC-7 gene positive isolates were sensitive to imipen- 
em. Cefepime and carbapenems were shown to be stable in response to these enzymes (6). In Acinetobacter spp., beta-lactamases are the most common factors creating resistance to imipenem and extended-spectrum cephalosporins, including extended-spectrum beta-lactamases (ESBL), carbapenem-hydrolyzing class D beta-lactamases (CHDL), Acinetobacter-derived cephalosporinases (ADC), and metallo-beta-lactamases (MBL) (17). Mobile genetic elements, may contribute to beta-lactamase overproduction and distribution (17).

The identification of imperative OXA-type carbapenemases and Acinetobacter-derived cephalosporinases in this study confirms the wide distribution of these enzymes in our hospital. An increase in the numbers of these enzymes may cause extended intra- and inter-hospital spread; therefore, it is necessary to design an effective protocol in order to control infections caused by $A$. baumannii which are resistant to carbapenems.

\section{Acknowledgements}

This research was supported by the Kashan University of Medical Sciences and the manuscript has been taken from a MS degree thesis (grant no: 88/6438).

\section{Financial Disclosure}

None declared.

\section{Funding/Support}

None declared.

\section{Authors' Contribution}

None declared.

\section{Ethical Consideration}

All ethical issues (such as informed consent, conflict of interest, plagiarism, misconduct,co-authorship, double submission, etc.) have been carefully considered.

\section{References}

1. Magnet S, Courvalin P, Lambert T. Resistance-nodulation-cell division-type efflux pump involved in aminoglycoside resistance in Acinetobacter baumannii strain BM4454. Antimicrob Agents Che- mother. 2001;45(12):3375-80.

2. Mak JK, Kim MJ, Pham J, Tapsall J, White PA. Antibiotic resistance determinants in nosocomial strains of multidrug-resistant Acinetobacter baumannii J Antimicrob Chemother. 2009;63(1):47-54.

3. Koh TH, Sng LH, Wang GC, Hsu LY, Zhao Y. IMP-4 and OXA beta-lactamases in Acinetobacter baumannii from Singapore. J Antimicrob Chemother. 2007;59(4):627-32.

4. Opazo A, Dominguez M, Bello H, Amyes SG, Gonzalez-Rocha G. OXA-type carbapenemases in Acinetobacter baumannii in South America. J Infect Dev Ctries. 2012;6(4):311-6.

5. Wikler MA. Performance standards for antimicrobial susceptibility testing: sixteenth informational supplement. 2006.

6. Hujer KM, Hamza NS, Hujer AM, Perez F, Helfand MS, Bethel CR et al. Identification of a new allelic variant of the Acinetobacter baumannii cephalosporinase, ADC-7 beta-lactamase: defining a unique family of class $C$ enzymes. Antimicrob Agents Chemother 2005;49(7):2941-8.

7. Hujer KM, Hujer AM, Hulten EA, Bajaksouzian S, Adams M, Donskey CJ, et al. Analysis of antibiotic resistance genes in multidrugresistant Acinetobacter sp. isolates from military and civilian patients treated at the Walter Reed Army Medical Center. Antimicrob Agents Chemother. 2006;50(12):4114-23.

8. Feizabadi MM, Fathollahzadeh B, Taherikalani M, Rasoolinejad M, Sadeghifard N, Aligholi M, et al. Antimicrobial susceptibility patterns and distribution of blaOXA genes among Acinetobacter spp. Isolated from patients at Tehran hospitals. Jpn J Infect Dis. 2008;61(4):274-8.

9. Dizbay M, Tunccan OG, Sezer BE, Hizel K. Nosocomial imipenemresistant Acinetobacter baumannii infections: epidemiology and risk factors . Scand J Infect Dis. 2010;42(10):741-6.

10. Brown S, Amyes S. OXA (beta)-lactamases in Acinetobacter: the story so far J Antimicrob Chemother. 2006;57(1):1-3.

11. Peleg AY, Seifert H, Paterson DL. Acinetobacterbaumannii: emergence of a successful pathogen. Clin Microbiol Rev. 2008;21(3):538-82.

12. Sohrabi N, Farajnia S, Akhi MT, Nahaei MR, Naghili B, Peymani A Prevalence of OXA-Type beta-Lactamases Among Acinetobacter baumannii Isolates from Northwest of Iran. Microb Drug Resist. 2012;18(4):385-9.

13. Evans BA, Hamouda A, Towner KJ, Amyes SG. OXA-51-like beta-lactamases and their association with particular epidemic lineages of Acinetobacter baumannii. Clin Microbiol Infect. 2008;14(3):268-75.

14. Merkier AK, Centron D. bla(OXA-51)-type beta-lactamase genes are ubiquitous and vary within a strain in Acinetobacter baumannii. Int J Antimicrob Agents. 2006;28(2):110-3.

15. Turton JF, Ward ME, Woodford N, Kaufmann ME, Pike R, Livermore DM, et al. The role of ISAba1 in expression of OXA carbapenemase genes in Acinetobacter baumannii. FEMS Microbiol Lett. 2006;258(1):72-7.

16. Corvec S, Caroff N, Espaze E, Giraudeau C, Drugeon H, Reynaud A. AmpC cephalosporinase hyperproduction in Acinetobacter baumannii clinical strains. J Antimicrob Chemother. 2003;52(4):629-35.

17. Huang LY, Lu PL, Chen TL, Chang FY, Fung CP, Siu LK. Molecular characterization of beta-lactamase genes and their genetic structures in Acinetobacter genospecies 3 isolates in Taiwan. Antimicrob Agents Chemother. 2010;54(6):2699-703. 